\title{
Ant Colony Optimization in Diverse Engineering Applications: an Overview
}

\author{
R. Geetha \\ Associate Professor, \\ Department of CSE, \\ S.A Engineering College \\ Chennai, Tamilnadu, India.
}

\author{
G. Umarani Srikanth \\ Associate Professor, \\ Department of PG Studies, \\ S.A Engineering College, \\ Chennai, Tamilnadu, India.
}

\begin{abstract}
Ant Colony Optimization (ACO) is a technique that was introduced in the early 1990's and it is inspired by the foraging behavior of ant colonies. ACO algorithms have long been thought as generating high quality solutions for various problems in different Engineering Applications. This survey provides an overview of past and on-going research of ACO in diverse engineering applications pertaining to computer science fields such as mobile and wireless networks, sensor networks, grid computing, P2P Computing, Pervasive computing, Data mining, Software engineering, Database systems, Multicore Processing, Artificial intelligence, Image processing, Biomedical applications and also other domains relevant to Electronics and Electrical Engineering fields. We finally summarize the comprehensive study of applications in all these fields deployed ACO.
\end{abstract}

\section{Keywords}

ACO, Mobile and Wireless Sensor Networks (WSNs), Grid and P2P computing, Pervasive Computing, Network Security, Data Mining, Multicore Processors, Engineering Applications.

\section{INTRODUCTION}

The growing importance of telecommunication and the Internet led to the development of more complex networked systems. The challenges of dealing with the vast complexity of networking problems such as handling large-scale networks, their dynamic nature, resource constraints, heterogeneity, load balancing, routing congestion control, unattended operation and robustness accentuate the need for more sophisticated techniques to solve these problems. Power consumption is one of the most important problems for WSNs because of the battery limitation in each sensor. Scheduling sensor activities is an effective way to prolong the lifetime of WSNs. The protection of integrity and confidentiality of the information and protection from unauthorized access are important issues. However due to factors such as resource limitations, absence of centralized access points, open wireless medium and small size of the sensor nodes, implementation of security in WSNs is a challenging task. Maximizing the lifetime of WSNs is also a challenging problem.

In mobile sensor networks [1], it is essential to manage the mobility of the nodes in order to improve the performance of the network. Location management is a complex problem in today's mobile computing environment. There is a need to but also the problem of human resource allocation. But as both of these two problems are difficult, existing models either suffer from a very large search space or have to restrict develop algorithms that could capture this complexity yet can be easily implemented and used to solve a wide range of location management scenarios.

It is a challenge for the traditional centralized or hierarchical grid architecture to manage the large-scale and dynamic resources while providing scalability. Task scheduling with load balancing in grid computing aims to assign tasks to computing nodes and minimize the execution time of tasks as well as workload across all nodes. The problem of scheduling workflows in terms of certain Quality of Service (QoS) requirements is challenging and it significantly influences the performance of grids. Grid service reliability, task scheduling

and resource discovery have not been sufficiently studied.

Constructing an efficient resource discovery mechanism is crucial for a decentralized and self-organized P2P information system in computational grids. How to reduce the number of messages or to improve searching efficiency has been a key problem which restricts the expanding of decentralized and unstructured Peer-to-Peer (P2P) networks. The principles of self-organization, resource searching and emergence have received a lot of interest in the research community recently.

Pervasive environments are becoming increasingly more popular due to the benefits of context-aware, user-centric service provisioning, despite their inherent challenges, i.e. dynamicity, heterogeneity and complexity. In such environments, multiple applications with distinct network requirements run simultaneously over the same underlying networks. Serving as abstractions to the latter, P2P overlays enable applications to operate over virtual networks that adhere to specified requirements.

The challenges within data mining research areas like discovering a list of classification rules, clustering, processing large amount of data and eliminate redundant information, etc need to be resolved in an intelligent manner. Feature selection and feature extraction are the two important processes in a classification system. High quality and fast clustering algorithms play a vital role for users to navigate, effectively organize and summarize data.

Research into developing effective computer aided techniques for planning software projects is also vital and challenging in software engineering. Different from projects in other fields, software projects are people-intensive activities and their related resources are mainly human resources. Thus an adequate model is required for software project planning has to deal with not only the problem of project task scheduling

the flexibility of human resource allocation to simplify the model. Resource allocation, as an integral part of Business Process Management (BPM), is more widely acknowledged 
by its importance for process-aware information systems. Despite the industrial need for efficient and effective resource allocation in BPM, few scientifically-grounded approaches exist to support these initiatives.

The problem of assigning each task in a given task graph to a processor in a reconfigurable multi processor architecture so as to minimize the total overall execution time of the tasks, information exchange in actual shared-memory parallel computers are some of the challenging research issues. The performance and the efficiency of a distributed database system depend highly on the way data are allocated to the sites. The NP-completeness of the data allocation problem and the large size of its real occurrence need a fast and scalable heuristic algorithm.

The reconstruction of DNA sequences from DNA fragments is one of the most challenging problems in computational biology. In recent years the specific problem of DNA sequencing by hybridization has attracted quite a lot of interest in the optimization community.

Modern digital systems consist of a complex mix of computational resources, e.g. microprocessors, memory elements and reconfigurable logic. System partitioning - the division of application tasks onto the system resources - plays an important role for the optimization of the latency, area, power and other performance metrics. The Semiconductor Wafer Fabrication System (SWFS) is extremely difficult to schedule due to its typical features such as large-scale, multiple re-entrant flows and hybrid machine types. Orthogonal Frequency Division Multiplexing (OFDM) is sensitive to Carrier Frequency Offset (CFO) that causes InterCarrier Interference (ICI).

Electrical-to-kinetic energy conversion efficiency of Synchronous Induction Coil Guns (SICG) is the main limiting factor of its development. In the system of SICG, the change of any electromagnetic parameters can directly or indirectly affects the electrical-to-kinetic energy conversion efficiency. Optimizing electric circuits with components, like resistors and capacitors, available in discrete values is a research issue. The solution to all the above mentioned issues is the use of bio inspired optimization technique called ACO which gives a quick and optimal solution to various problems related to different Engineering Applications.

\section{ANT COLONY OPTIMIZATION}

ACO [2] is an algorithm based on the behavior of the real ants in finding a shortest path from a source to the food. It utilizes the behavior of the real ants while searching for the food. It has been observed that the ants deposit a certain amount of pheromone in its path while traveling from its nest to the food. Again while returning, the ants are subjected to follow the same path marked by the pheromone deposit and again deposit the pheromone in its path. In this way the ants following the shorter path are expected to return earlier and hence increase the amount of pheromone deposit in its path at a faster rate than the ants following a longer path.

ACO takes the inspiration from the foraging behavior of the ants. These ants deposit pheromone on the ground in order to mark some favorable path that should be followed by other members of the colony. However the pheromone is subjected to evaporation by a certain amount at a constant rate after a certain interval and therefore the paths visited by the ants frequently are only kept as marked by the pheromone deposit, whereas the paths rarely visited by the ants are lost because of the lack of pheromone deposit on that path and as a result the new ants are intended to follow the frequently used paths only. Thus all the ants starting their journey can learn from the information left by the previously visitor ants and are guided to follow the shorter path directed by the pheromone deposit. In ACO a number of artificial ants(here data packets) build solutions to the considered optimization problem at hand and exchange information on the quality of these solutions via a communication scheme that is pheromone deposit on the path of the journey performed by it. ACO has been formalized into a metaheuristic [3] for combinatorial optimization problems by Dorigo.

\section{APPLICATIONS OF ACO}

ACO is being widely used in different Engineering Applications. This section addresses few recent issues which are solved by various authors in various fields of Engineering such as Computer Science, Electrical, Electronics, and Biomedical etc. using Ant Colony Optimization Techniques.

\subsection{Mobile and Wireless Sensor Networks (WSNs)}

In mobile sensor networks, it is important to manage the mobility of the nodes in order to improve the performances of the network. The problem of single target tracking in controlled mobility sensor networks is addressed by the author [1]. The proposed method consists of estimating the current position of a single target. Estimated positions are then used to predict the following location of the target. While the estimation and the prediction phases are performed using the interval theory, relocating nodes employs ACO. Simulations results corroborate the efficiency of the method compared to the target tracking methods considered for networks with static nodes.

Maximizing the lifetime of WSNs is a challenging problem. The methodology is based on finding the maximum number of disjoint connected covers that satisfy both sensing coverage and network connectivity. The approach using ACO [4] has been applied to a variety of heterogeneous WSNs. The results show that the approach is effective in finding high-quality solutions for maximizing the lifetime of heterogeneous WSNs.

The author proposed the ACO approach to distributed Sensor Wakeup Control (SWC) in WSNs [5] to accomplish the joint task of surveillance and target tracking or cluster leaders. The approach is free of the problems caused by leader failures and can save the communication cost for leader selection. It is robust to false alarms because the pheromone is accumulated temporally and spatially, thus is more reliable for wakeup control. The method does not need the knowledge of node position. Simulations are carried out to evaluate the performance of the method in comparison with representative methods.

ACO mechanisms and the associated techniques for resolving routing in WSNs are investigated in [6]. The author presented a mathematical theory of the biological computations in the context of sensor networks and a generalized routing framework in sensor networks by diffusing different modes of biological computations using ACO and genetic approaches.

\subsection{Network Security}

Applying the idea of ACO into network vulnerability detection and restoration system can improve the performance of network security management. A novel method is proposed in [7] called Multi mobile Agent Vulnerability Detection and Restoration System (MAVDRS) in which the restoration mechanism which adopts ACO is proposed. It is proved that the model can ensure Intranet's security has a lower delay and higher network bandwidth utilization. 


\subsection{Peer To Peer (P2P) and Grid Computing}

A new trust evaluation model based on ACO is presented in [8]. The recommendation relationship and the pheromone are associated in this model; several optimized trust paths between the requesting peer and the target peer are archived by enforcing the extended ACO Algorithm. The reputation value of the target peer is composed of the trust values generated from different recommendation paths and the requesting peers always select the target peers with uppermost reputation values for transaction. It is substantiated that this model can further alleviate negative impact due to the malicious peers and reduce the message overhead.

In [9] Antares which is a bio-inspired algorithm for the construction of a decentralized and self-organized P2P information system in computational grids is addressed. This self-structured organization combines the benefits of both unstructured and structured P2P information systems. Indeed, being basically unstructured, Antares is easy to maintain in a dynamic grid, in which joins and departs of hosts can be frequent events. On the other hand, the aggregation and spatial ordering of descriptors can improve the rapidity and effectiveness of discovery operations which is a beneficial feature typical of structured systems. Performance analysis proved that ant operations allow the information system to be efficiently reorganized thus improving the efficacy of both simple and range queries.

In grid environment discussed in [10] applications are always regarded as workflows. The problem of scheduling workflows in terms of certain QoS requirements is challenging and it significantly influences the performance of grids. The ACO algorithm proposed schedules large-scale workflows with various QoS parameters. This algorithm enabled users to specify their QoS preferences as well as to define the minimum QoS thresholds for a certain application. The objective of this algorithm is to find a solution that meets all QoS constraints and optimizes the user-preferred QoS parameter. Based on the characteristics of workflow scheduling, the author has designed seven new heuristics for the ACO approach and proposed an adaptive scheme that allows artificial ants to select heuristics based on pheromone values. Experiments are done in ten workflow applications with at most 120 tasks and the results demonstrate the effectiveness of the proposed algorithm.

By integrating the P2P philosophy and techniques into a Grid architecture, P2P Grid system is emerging as a promising platform for executing large-scale, resource intensive applications. The author in [11] proposed and designed a large-scale P2P Grid system which employs an ACO algorithm to locate the required resources. The ACO method avoids a large-scale flat flooding and supports multi-attribute range query. Multiple ants can be employed to improve the parallelism of the method. Comprehensive simulation results validated the effectiveness of the proposed method compared with the traditional unstructured and structured approaches.

For some grid services which have large subtasks requiring time-consuming computation, the reliability of grid service could be rather low. To resolve this problem, the author in [12] introduced Local Node Fault Recovery (LNFR) mechanism into grid systems and presented an in-depth study on grid service reliability modeling and analysis with this kind of fault recovery. To make LNFR mechanism practical, some constraints, i.e. the life times of subtasks and the numbers of recoveries performed in grid nodes are introduced and grid service reliability models under these practical constraints are developed. Based on the proposed grid service reliability model the author presented a multi-objective task scheduling optimization model and developed ACO algorithm to solve it effectively.

\subsection{Pervasive Computing}

Pervasive environments are becoming increasingly popular due to the benefits of context-aware, user-centric service provisioning, despite their inherent challenges, i.e. dynamicity, heterogeneity and complexity. In such environments, multiple applications with distinct network requirements run simultaneously over the same underlying networks. Serving as abstractions to the latter, P2P overlays enable applications to operate over virtual networks that adhere to specified requirements. The author in [13] proposed AntOM, a topology optimization algorithm for multi-layer $\mathrm{P} 2 \mathrm{P}$ overlays on top of pervasive environments. The algorithm is inspired by ACO in regards to the network exploration and neighborhood optimization. By utilizing different ant families, multiple overlay layers, each one optimized for a different property, can be concurrently maintained at a low cost as proven by the experimental results.

\subsection{Computer Architecture}

The author in [14] combined the memory principle and ACO in Intrusion Detection System (IDS). The method of controlling pheromone used in ACO is applied to simulate the memory process of human brain and the concept of pheromone is put forth. The processes of memorizing and forgetting are reasonably interpreted through the increase and decrease of pheromone in the algorithm, a new memory algorithm is formed to finish the processes of memorizing and forgetting after considering the impact of abnormal characteristics weight value on pheromone. Examples show that the algorithm is capable of realizing memorizing and forgetting processes and increasing the robustness and self adaptability of IDS.

The problem of determining whether a set of periodic tasks can be assigned to a set of heterogeneous processors without deadline violations has been shown, in general, to be NP-hard. The author in [15] presented a new algorithm based on ACO meta-heuristic for solving this problem. A local search heuristic that can be used by various meta-heuristics to improve the assignment solution is proposed and its time and space complexity is analyzed. In addition to being able to search for a feasible assignment solution, this extended ACO algorithm can optimize the solution by lowering its energy consumption.

\subsection{Data Mining}

The author in [16] proposed a new sequential covering strategy for ACO classification algorithms to mitigate the problem of rule interaction, where the order of the rules is implicitly encoded as pheromone values and the search is guided by the quality of a candidate list of rules. It is experimented using eighteen publicly available data sets and proved that the predictive accuracy obtained by a new ACO classification algorithm implementing the sequential covering strategy is statistically significantly higher than the predictive accuracy of state-of-the-art rule induction classification.

The author in [17] created a system for power load forecasting using support vector machine and ACO. A method is employed to process large amount of data and eliminate redundant information. The system mines the historical daily loading which has the same meteorological category as the forecasting day in order to compose data sequence with highly similar meteorological features. The method reduced Support Vector Machine (SVM) training data and overcome the disadvantage of very large data and slow processing speed 
when constructing SVM model. It can mine the data more overall and accurate than the original fuzzy-rough method, an entropy-based feature selector, and a transformation-based reduction method, PCA. It is proved that the new method can achieve greater forecasting accuracy when compared with single SVM and BP neural network in short-term load forecasting

\subsection{Image Processing}

An edge detection technique based on ACO which established a pheromone matrix that represents the edge information at each pixel based on the routes formed by the ants dispatched on the image is addressed [18]. The movement of the ants is guided by the local variation in the image's intensity values. The proposed method takes advantage of the improvements introduced in ant colony system, one of the main extensions to the original ant system. Experimental results proved the success of the technique in extracting edges from a digital image.

The author in [19] proposed a novel scheme for texture segmentation and representation based on ACO. Texture segmentation and texture characteristic expression are two important areas in image pattern recognition. Nevertheless, until now, how to find an effective way for accomplishing these tasks is still a major challenge in practical applications such as iris image processing. By defining different kinds of direction probability and movement difficulty for artificial ants, an ACO based image segmentation algorithm and a texture representation method are presented for automatic iris image processing. Experimental results demonstrated that the ACO based image processing methods are competitive and quite promising, with excellent effectiveness and practicability especially for images with complex local texture situations.

The estimation of noise statistics is critical to optimize many computer vision algorithms. The main issue is how to identify the homogeneous image patches for estimating the noise statistics. The author in [20] proposed an approach that has two key components. First, a graphical representation is proposed to model the relationship among image patches. Second, the ACO technique is used to automatically select a set of patches for estimating the noise statistics. To be more specific, this approach guided the spatial movement of artificial ants towards homogeneous locations in the graph, by considering both global (i.e., clustering measure) properties and local (i.e., homogeneity measure) properties of patches. It is proved that this approach outperforms nine conventional approaches to provide more accurate noise statistics estimation.

\subsection{Software Engineering}

The Multimode Resource-Constrained Project Scheduling Problem with Discounted Cash Flows (MRCPSPDCF) is challenging for project management. To design an effective algorithm for the MRCPSPDCF, the author in [21] proposed an ACO based approach. It is promising for the MRCPSPDCF due to the following three reasons. First, MRCPSPDCF can be formulated as a graph-based search problem, which ACO has been found to be good at solving. Second, the mechanism of ACO enables the use of domain-based heuristics to accelerate the search. Furthermore ACO has found good results for the classical single-mode scheduling problems. This algorithm is compared with two different Genetic Algorithms (GAs), a Simulated Annealing (SA) algorithm and a Tabu Search (TS) algorithm on 55 random instances. Experimental results proved that the algorithm outperforms the GA, SA, and TS approaches on most cases.
Research into developing effective computer aided techniques for planning software projects is important and challenging for software engineering. Thus an adequate model for software project planning has to deal with not only the problem of project task scheduling but also the problem of human resource allocation. To develop a flexible and effective model for software project planning, the author in [22] proposed a novel approach with an Event-Based Scheduler (EBS) and an ACO algorithm. The approach represents a plan by a task list and a planned employee allocation matrix. In this way, both the issues of task scheduling and employee allocation can be taken into account. In the EBS, the beginning time of the project, the time when resources are released from finished tasks and the time when employees join or leave the project are regarded as events. The basic idea of the EBS is to adjust the allocation of employees at events and keep the allocation unchanged at non-events. With this strategy, the proposed method enabled the modeling of resource conflict and task preemption and preserves the flexibility in human resource allocation.

Network analysis provides an effective practical system for planning and controlling large projects in construction and many other fields. The author in [23] presented the use of ACO system for solving and calculating both deterministic and probabilistic Critical Path Method/ Programme Evaluation Review Technique (CPM/PERT) networks. This method is investigated for a selected case study in construction management. The results demonstrated that ACO can produce good optimal and suboptimal solutions.

\subsection{Electronics}

The author in [24] introduced ACO into the design of flatgain-spectrum Raman Fiber Amplifiers (RFAs) for the first time. In this novel design method, the pump powers and pump wavelengths are optimized by ACO to obtain a flat gain spectrum with a pre-specified maximum gain ripple under the given parameters of RFAs. The author proved that the proposed method is efficient and stable.

The author in[25] proposed ACO application to a Fuzzy Controller (FC) design, called ACO-FC for improving design efficiency and control performance as well as ACO hardware implementation. An FC's antecedent part, i.e., the "if" part of its composing fuzzy if-then rules is partitioned in grid-type and all candidate rule consequent values are then listed. An ant trip is regarded as a combination of consequent values selected from every rule. A pheromone matrix among all candidate consequent values is constructed. Searching for the best one among all combinations of rule consequent values is based mainly on the pheromone matrix. The author proved that ACO-FC performance is better than other meta-heuristic design methods on simulation examples.

\subsection{Electrical}

Electrical-to-kinetic energy conversion efficiency of Synchronous Induction Coil Guns (SICG) is the main limiting factor of its development. In the system of SICG, the change of any electromagnetic parameters can directly or indirectly affects the electrical-to-kinetic energy conversion efficiency. In order to improve the electrical-to-kinetic energy conversion efficiency of SICG, the electromechanical model of SICG was built. The author in [26] optimized the structural parameters of SICG with $60-\mathrm{mm}$ caliber with ACO. The electrical-tokinetic energy conversion efficiency of the system was regarded as the target function, while the center-to-center space between the drive coil and the armature and the structural parameters of the drive coil and the armature were regarded as variables. Results of research indicated that the 
electrical-to-kinetic energy conversion efficiency of the system was improved through parameter optimization.

\subsection{Biomedical}

The reconstruction of DNA sequences from DNA fragments is one of the most challenging problems in computational biology. In recent years the specific problem of DNA sequencing by hybridization has attracted quite a lot of interest in the optimization community. Several metaheuristics such as TS and evolutionary algorithms have been applied to this problem. However, the performance of existing meta-heuristics is often inferior to the performance of recently proposed constructive heuristics. On the basis of these new heuristics the author in [27] developed an ACO algorithm for DNA sequencing by hybridization. An important feature of this algorithm is the implementation in a so-called multi-level framework. The computational results proved that this algorithm is currently a state-of-the-art method for the tackled problem.

A Hyper-box clustering with Ant Colony Optimization (HACO) is addressed in [28] for classifying unlabeled data using hyper-boxes. c. It acknowledged the topological information of the data while looking in a small search space, providing results with high precision in a short time. It is validated using artificial $2 \mathrm{D}$ data sets and then applied to a real medical data set, automatically extracting medical risk profiles, a laborious operation for doctors. Clustering results show an improvement of $36 \%$ in accuracy and 7 times faster processing time when compared to the usual ant colony optimization approach. It can be further extended to hyperbox shape optimization (fine tune accuracy), automatic parameter setting (improve usability) and applied to diagnosis decision support systems.

\subsection{Summary of the ACO applications}

Apart from these applications ACO also addresses the problems like Traveling Salesman Problem (TSP), Vehicle routing, Sequential ordering, Quadratic assignment, Course timetabling, Graph coloring, Car sequencing, Multiple knapsack. Table 1 summarizes the different applications of ACO which are explained in section 3.

Table 1: Summary of the ACO applications

\begin{tabular}{|c|c|c|}
\hline Domain & Type of the problem solved & Advantages of the Solved Problem \\
\hline \multirow[t]{4}{*}{$\begin{array}{l}\text { Mobile and } \quad \text { Wireless } \\
\text { Sensor Networks }\end{array}$} & Target Tracking & $\begin{array}{l}\text { Efficient when compared to the target tracking methods considered } \\
\text { for networks with static nodes. }\end{array}$ \\
\hline & $\begin{array}{l}\text { Maximizing the lifetime of } \\
\text { nodes }\end{array}$ & Applied to a variety of heterogeneous WSNs \\
\hline & Sensor wakeup control & $\begin{array}{l}\text { free of the problems caused by leader failures and can save the } \\
\text { communication cost for leader selection }\end{array}$ \\
\hline & Routing & Low Routing Overhead \\
\hline Network Security & $\begin{array}{l}\text { Vulnerability Detection and } \\
\text { Restoration }\end{array}$ & Lower delay and higher network bandwidth utilization. \\
\hline \multirow{5}{*}{$\begin{array}{l}\text { Peer-to-Peer(P2P) \& Grid } \\
\text { Computing }\end{array}$} & Trust Evaluation of the Peers & Alleviate the negative impact due to the malicious peers \\
\hline & $\begin{array}{l}\text { Self-organized P2P information } \\
\text { System construction }\end{array}$ & $\begin{array}{l}\text { Information system is efficiently reorganized, thus improving the } \\
\text { efficacy of both simple and range queries. }\end{array}$ \\
\hline & Scheduling Workflows (QoS) & $\begin{array}{l}\text { Effective for more than ten workflow applications with at most } 120 \\
\text { tasks. }\end{array}$ \\
\hline & Locating the required resources & $\begin{array}{l}\text { Avoids a large-scale flat flooding and supports multi-attribute range } \\
\text { query }\end{array}$ \\
\hline & Grid Service reliability & Fault recovery on grid service reliability is efficient. \\
\hline Pervasive Computing & Topology Optimization & $\begin{array}{l}\text { Low Cost (Applications with diverse requirements can be served at } \\
\text { the same time by exploiting the same network infrastructure.) }\end{array}$ \\
\hline Computer Architecture & Task Assignment & $\begin{array}{l}\text { Feasible task assignment solution and lowers the energy } \\
\text { consumption }\end{array}$ \\
\hline \multirow[t]{2}{*}{ Data Mining } & Classification algorithm. & $\begin{array}{l}\text { Predictive accuracy obtained is statistically significantly higher than } \\
\text { the predictive accuracy of state-of-the-art rule induction } \\
\text { classification. }\end{array}$ \\
\hline & $\begin{array}{l}\text { Power load forecasting using } \\
\text { support vector machine }\end{array}$ & $\begin{array}{l}\text { Greater forecasting accuracy when compared with single SVM and } \\
\text { BP neural network in short-term load forecasting }\end{array}$ \\
\hline \multirow[t]{3}{*}{ Image Processing } & Edge Detection & Efficient in extracting edges from a digital image. \\
\hline & texture segmentation & $\begin{array}{l}\text { Effectiveness and practicability especially for images with complex } \\
\text { local texture situations. }\end{array}$ \\
\hline & Image Noise Estimation & Provides more accurate noise statistics estimation. \\
\hline
\end{tabular}




\begin{tabular}{|c|c|c|}
\hline \multirow[t]{3}{*}{ Software Engineering } & Software Project Management & Outperforms the GA, SA and TS approaches on most cases. \\
\hline & $\begin{array}{l}\text { Software Project Scheduling } \\
\text { And Staffing }\end{array}$ & $\begin{array}{l}\text { Enables the modeling of resource conflict and task preemption and } \\
\text { preserves the flexibility in human resource allocation. }\end{array}$ \\
\hline & CPM/PERT networks & $\begin{array}{l}\text { ACO can produce good optimal and suboptimal solutions compared } \\
\text { to conventional methods. }\end{array}$ \\
\hline \multirow[t]{2}{*}{ Electronics } & $\begin{array}{l}\text { Design of flat-gain-spectrum } \\
\text { Raman fiber amplifiers }\end{array}$ & The proposed method is efficient and stable \\
\hline & Fuzzy Controller Design & Improves the design efficiency and control performance of the chip. \\
\hline Electrical & $\begin{array}{l}\text { Synchronous Induction Coil } \\
\text { guns. }\end{array}$ & $\begin{array}{l}\text { Efficiency of the system was improved through parameter } \\
\text { optimization. }\end{array}$ \\
\hline \multirow[t]{2}{*}{ Bio medical } & $\begin{array}{l}\text { Reconstruction of DNA } \\
\text { sequences from DNA fragments }\end{array}$ & Currently a state-of-the-art method for the tackled problem. \\
\hline & $\begin{array}{l}\text { Medical Risk Profile } \\
\text { Recognition }\end{array}$ & Provides results with high precision in a short time \\
\hline
\end{tabular}

\section{CONCLUSION}

In this paper we have given a brief overview of the applications of ACO in various engineering domains. Not only in the above mentioned fields, is ACO also used in many industrial applications. Study of those applications can also be explored. ACO can also be applied to various unsolved issues in the emerging fields of Engineering.

\section{ACKNOWLEDGEMENT}

We thank the management and Principal of S.A Engineering College for giving us constant motivation in enhancing our technical skills.

\section{REFERENCES}

[1] Farah Mourad, Hicham Chehade, Hichem Snoussi, Farouk Yalaoui, Lionel Amodeo, C'Edric Richard "Controlled Mobility Sensor Networks For Target Tracking Using Ant Colony Optimization", IEEE Transactions On Mobile Computing, Volume 11, pp: $1261-1273,2011$

[2] M. Dorigo, V. Maniezzo \& A. Colorni, "Ant System: Optimization by A Colony of Cooperating Agents", IEEE Transactions on Systems, Man, and CyberneticsPart B, vol 26, pp: 29-41, 1996

[3] Marco Dorigo, Mauro Birattari, and Thomas Stutzle, "Ant Colony Optimization Artificial Ants as a Computational Intelligence Technique", IEEE Computational Intelligence Magazine, 2006

[4] Ying Lin, Jun Zhang, Henry Shu-Hung Chung Wai Hung Ip, Yun Li, and Yu-Hui Shi, "An Ant Colony Optimization Approach for Maximizing the Lifetime of Heterogeneous Wireless Sensor Networks", IEEE Transactions on Systems, Man and Cybernetics-Part C: Applications and Reviews, 2011

[5] Yan Liang, Jiannong Cao, Lei Zhang, Rui Wang, Quan Pan, "A Biologically Inspired Sensor Wakeup Control Method For Wireless Sensor Networks", IEEE Transactions On Systems, Man, And Cybernetics-Part C: Applications And Reviews, Vol. 40, 2010
[6] Iyengar S. S., Hsiao-Chun Wu, N. Balakrishnan, and Shih Yu Chang, "Biologically Inspired Cooperative Routing For Wireless Mobile Sensor Networks", IEEE Systems Journal, Vol. 1, 2007

[7] Wu Min, Zhang Zhi-Ming "Using Ant Colony Optimization to Modeling the Network Vulnerability Detection and Restoration System", International Conference OnIndustrial Mechatronics and Automation, 2009, ICIMA 2009

[8] Lei Yang; Zhiguang Qin; Can Wang; Yao Liu; Chaosheng Feng, "A P2P Reputation Model Based On Ant Colony Algorithm", International Conference on Communications, Circuits and Systems, 2010

[9] Abortion Forestiero, And Carlo Mastroianni, "A Swarm Algorithm for a Self-Structured P2P Information System", IEEE Transactions on Evolutionary Computation, Vol. 13, 2009

[10] Wei-Neng Chen, Jun Zhang, “An Ant Colony Optimization Approach To A Grid Workflow Scheduling Problem With Various Qos Requirements", IEEE Transactions On Systems, Man, and Cybernetics,Vol-39, 2009

[11] Yuhui Deng, Frank Wang, Adrian Ciura, "Ant Colony Optimization Inspired Resource Discovery In P2P Grid Systems", The Journal of Supercomputing, Volume 49, 2009

[12] Suchang Guo, Hong-Zhong Huang, Zhonglai Wang, and Min Xie, "Grid Service Reliability Modeling and Optimal Task Scheduling Considering Fault Recovery", IEEE Transactions on Reliability, Vol. 60, 2011

[13] Peng, Fei; Malatras, Apostolos; Hirsbrunner, Boat; Courant, Michele, "Antom: Constructing Multi-Layer Overlays for Pervasive Environments", IEEE International Conference on Pervasive Computing and Communications Workshops (PERCOM Workshops), 2012

[14] Yong Liu; Sunjun Liu, "Intrusion Detection System Based On Ant Colony Memory Principle", International Conference On Apperceiving Computing And Intelligence Analysis, 2009. 
[15] Hua Chen , Albert Mo Kim Cheng, Ying-Wei Kuo, "Assigning Real-Time Tasks To Heterogeneous Processors By Applying Ant Colony Optimization", Journal Of Parallel and Distributed Computing, Vol 71, 2011

[16] Otero, F., Freitas, A. Johnson, C. “A New Sequential Covering Strategy for Inducing Classification Rules With Ant Colony Algorithms", 2012

[17] Dongxiao Niu, Yongli Wang, Desheng Dash Wu, "Power Load Forecasting Using Support Vector Machine And Ant Colony Optimization", An International Journal On Expert Systems With Applications, 2010

[18] Anna Veronica Baterina, Carlos Oppus , "Image Edge Detection Using Ant Colony Optimization”, WSEAS Transactions on Signal Processing, Vol 6, 2010

[19] Lin Ma, Kuanquan Wang, David Zhang, "A Universal Texture Segmentation And Representation Scheme Based On Ant Colony Optimization For Iris Image Processing", International Journal Of Computers \& Mathematics with Applications, Vol 57, 2009

[20] Jing Tian and Li Chen, "Image Noise Estimation Using a Variation-Adaptive Evolutionary Approach", IEEE Signal Processing Letters, Vol. 19, 2012

[21] Wei-Neng Chen, Jun Zhang, Henry Shu-Hung Chung, Rui-Zhang Huang, Ou Liu, "Optimizing Discounted Cash Flows In Project Scheduling: An Ant Colony Optimization Approach", IEEE Transactions On Systems, Man, And Cybernetics, Part C: Applications And Reviews, Vol 40, 2010

[22]Wei-Neng Chen and Jun Zhang, “Ant Colony Optimization for Software Project Scheduling and
Staffing With an Event-Based Scheduler", IEEE Transactions on Software Engineering, Vol 99, 2012

[23] Hazem Abdallah, Hassan M. Emara, Hassan T. Dorrah, Ahmed Bahgat, "Using Ant Colony Optimization Algorithm For Solving Project Management Problems", Expert Systems With Applications: An International Journal, Vol 36,2009

[24] Hai Ming Jiang, Kang Xie and Ya Fei Wang, "Novel Design of Flat Gain Spectrum Raman Fiber Amplifiers Based On Ant Colony Optimization", IEEE Photonics Technology Letters, Vol. 23, 2011

[25] Chia-Feng Juang, Chun-Ming Lu, Chiang Lo and ChiYen Wang, "Ant Colony Optimization Algorithm for Fuzzy Controller Design and Its FPGA Implementation", IEEE Transactions on Plasma Science, Vol. 39, 2011

[26] Wenbiao Liu, Cao Yanjie, Yuan Zhang, Jie Wang and Dawei Yang, "Parameters Optimization of Synchronous Induction Coilgun Based On Ant Colony Algorithm", IEEE Transactions on Plasma Science, Vol. 39, 2011

[27] Christian Blum, Mateu Yábar Vallès, Maria J. Blesa , "An Ant Colony Optimization Algorithm For DNA Sequencing By Hybridization", Journal Computers And Operations Research, Vol 35, pp:3620-3635, 2008

[28] G. N. Ramos, Y. Hatakeyama , F. Dong, K. Hirota , "Hyperbox Clustering With Ant Colony Optimization (HACO) Method And Its Application To Medical Risk Profile Recognition", Journal Of Applied Soft Computing, Vol 9, 2009 